\title{
Anomalous coronary arteries: Depiction at dual-source computed tomographic coronary angiography
}

\author{
Hai Xu, MD, Yinsu Zhu, MD, Xiaomei Zhu, MD, Lijun Tang, MD, PhD, and Yi Xu, MD
}

Objective: To retrospectively determine the imaging features of coronary artery anomalies depicted at dual-source computed tomographic coronary angiography (DSCT-CA).

\begin{abstract}
Methods: We reviewed the case histories of 12,145 patients with suspected coronary arterial disease who underwent DSCT-CA at our institution. Multiplanar reformation, maximum-intensity projection, and volumerendered imaging were performed on an offline workstation. Each study was assessed retrospectively for the origin and course of the anomalous coronary artery by a minimum of 2 cardiovascular radiologists; decisions were made in consensus.
\end{abstract}

Results: There were 124 (1.02\%) patients with coronary anomalies. Fifty-one patients demonstrated an anomalous origin of the right coronary artery from the left sinus of Valsalva or the left main artery. An anomalous origin of a left circumflex artery from the right sinus of Valsalva or the right coronary artery was depicted in 17 patients. An anomalous origin of a left main artery from the right sinus of Valsalva was depicted in 1 patient. A single coronary artery was shown in 4 patients, and congenital transposition of the great arteries was associated with this anomaly in 1 patient. In the remaining 50 patients, coronary artery fistulas were identified. Eight patients were referred after an equivocal conventional coronary angiogram.

Conclusions: DSCT-CA is a reliable noninvasive tool that allows accurate delineation of coronary arterial anomalies in an appropriate clinical setting and provides detailed 3-dimensional anatomic information that may be difficult to obtain with invasive coronary angiography. (J Thorac Cardiovasc Surg 2012;143:1286-91)

Coronary artery anomalies are rare. Most of these anomalies produce no clinical symptoms during life. Approximately $20 \%$ of these anomalies produce life-threatening symptoms, including arrhythmias, syncope, myocardial infarction, or sudden death. ${ }^{1}$ Indeed, congenital coronary artery anomalies are the common cause of sudden death in young competitive athletes, accounting for $13 \%$ of cases, which is second in frequency to hypertrophic cardiomyopathy. ${ }^{2,3}$

For several decades, conventional invasive coronary angiography has been used for premorbid diagnosis of coronary artery anomalies. ${ }^{4}$ However, this technique is not only invasive, but expensive, and cannot always adequately provide the precise information about the abnormal vessels. ${ }^{1}$ Noninvasive coronary angiography has become feasible as temporal and spatial resolution improves with the development of electrocardiographically gated multi-

From the Department of Radiology, the First Affiliated Hospital of Nanjing Medical University, Nanjing, Jiangsu, China.

Disclosures: Authors have nothing to disclose with regard to commercial support.

Drs Hai Xu and Yinsu Zhu contributed equally to this work.

Received for publication Sept 6, 2011; revisions received Oct 20, 2011; accepted for publication Nov 9, 2011; available ahead of print Dec 12, 2011.

Address for reprints: Lijun Tang, MD, PhD, The First Affiliated Hospital of Nanjing Medical University, Department of Radiology, 300 Guangzhou Rd, Nanjing, Jiangsu, 210029, China (E-mail: Lijun.tang@hotmail.com); or Yi Xu, MD, The First Affiliated Hospital of Nanjing Medical University, Department of Radiology, 300 Guangzhou Rd, Nanjing, Jiangsu, 210029, China. (E-mail: aleenxu@ hotmail. com).

0022-5223/\$36.00

Copyright $($ c 2012 by The American Association for Thoracic Surgery doi:10.1016/j.jtcvs.2011.11.025 detector row computed tomography (MDCT). ${ }^{5}$ However, MDCT imaging can be limited by motion artifacts caused by limited temporal resolution in patients with high heart rates as well as patients with arrhythmia.

The recently introduced dual-source computed tomography (DSCT) scanner uses $2 \mathrm{x}$-ray tubes and detectors to achieve an improved temporal resolution up to $83 \mathrm{~ms}$. It is now possible and practical to have high-quality cardiac imaging without beta-blocker premedication even in patients with high heart rates. 6,7

The aim of this article is to retrospectively determine the imaging features of coronary artery anomalies depicted at DSCT coronary angiography (DSCT-CA).

\section{PATIENTS AND METHODS \\ Patients}

Between July 2006 and June 2011, 12,415 case studies of DSCT-CA were conducted in our institution. All patients had known or suspected coronary artery obstructive disease with atypical chest pain or stable angina. Contraindications for the examination were previous allergy to iodinated contrast medium, nonsinus rhythm, renal insufficiency, and cardiac insufficiency. A retrospective evaluation was performed to identify all patients with anomalous coronary vessels.

\section{DSCT-CA: Scan Protocol and Reconstruction}

All patients were examined with a DSCT system (Somatom Definition; Siemens Medical Systems, Erlangen, Germany). Before scanning was performed, the procedure was explained to the patients in detail and nitroglycerin $(0.5 \mathrm{mg})$ was administered sublingually to induce coronary vasodilatation. Between 40 and $110 \mathrm{~mL}$ of nonionic contrast medium (Schering Ultravist, Iopromide, $370 \mathrm{mg}$ iodine per milliliter) was injected 


$$
\begin{array}{ll}
\text { Abbreviations and Acronyms } \\
\text { DSCT = } \\
\text { DSCT-CA = } & \text { dual-source computed tomography } \\
& \text { coronary angiography } \\
\text { MDCT }= & \text { multi-detector row computed } \\
& \text { tomography } \\
\text { MIP }= & \text { maximum-intensity projection } \\
\text { MPR }= & \text { multiplanar reformation } \\
\text { MR }= & \text { magnetic resonance } \\
\text { VR } & =\text { volume rendered }
\end{array}
$$

intravenously at a flow rate of 2.3 to $7.4 \mathrm{~mL} / \mathrm{s}$ followed by a $40-\mathrm{mL}$ saline flush at the same rate of contrast. Beta-blocker premedication was not used in any patient before the DSCT examination for heart rate regulation.

Bolus tracking was used to trigger data acquisition by placing a region of interest over the descending aorta and setting the trigger threshold to 100 Hounsfield units above baseline. Scanning was performed in a craniocaudal direction covering the region from the aortic root above the ostium of the left coronary artery to the diaphragm. Scanning parameters were as follows: detector collimation, $2 \times 32 \times 0.6 \mathrm{~mm}$; slice collimation, $2 \times 64 \times 0.6 \mathrm{~mm}$ by means of a z-flying focal spot; gantry rotation time, $330 \mathrm{~ms}$; heart rate-dependent pitch, 0.20 to 0.42 ; tube current, 380 to $400 \mathrm{mAs}$; tube voltage, 120 $\mathrm{kV}$. The scan time was about 7.9 to 14.1 seconds for a single breath hold.

The coronary arteries were reconstructed retrospectively at best diastolic or best systolic phases with the fewest motion artifacts. Axial images were reconstructed with $0.75-\mathrm{mm}$ slice thicknesses and 0.5 - $\mathrm{mm}$ increments using a medium soft-tissue convolution kernel (B26). Further reconstructions were obtained with 2-dimensional multiplanar reformation (MPR), 3-dimensional maximum-intensity projection (MIP), and 3-dimensional volume-rendered (VR) imaging were performed on an offline workstation (Syngo Multimodality Workstation; Siemens, Erlangen, Germany).

\section{Image Review}

All images were assessed in a blinded manner by a minimum of 2 cardiovascular radiologists. The determination of coronary artery origin and course of the anomalous vessel was reached by consensus.

\section{RESULTS}

DSCT-CA was performed successfully without any complication in all patients. There were 124 patients ( 80 male and 44 female) with coronary anomalies. The patients ranged in age from 5 to 86 years (mean age, 57.6 years). The effective radiation dose ranged from 10.5 to 22.3 $\mathrm{mSv}$ (mean dose, $13.6 \mathrm{mSv}$ ). The heart rate ranged from 45 to 126 beats/min (mean heart rate, 81 beats/min).

Among the $80(64.5 \%)$ men and $44(35.5 \%)$ women, there were 69 anomalies with origin of the coronary artery or branch from the opposite or noncoronary sinus and an anomalous course (Figures 1, $A$ and $B$, and 2), 4 anomalies with a single coronary artery (Figure $1, C$ ), 1 anomaly associated with congenital transposition of the great arteries, and 50 anomalies with coronary artery fistula (Figure 3). In 1 of these, a complex coronary artery-pulmonary artery fistula with conus artery aneurysm was clearly delineated using a 3-dimensional VR image (Figure 3,B), which was misdiagnosed as a thrombus in the right atrium by echocardiography.

Incidences of coronary anomalies and patterns were summarized in Table 1. Origin and drainage sites of coronary artery fistulas in 50 patients were summarized in Table 2.

Among 69 patients with anomalous origin of the coronary artery from the opposite sinus, 50 anomalous arteries ran between the aorta and the pulmonary artery and took an interarterial course (Figures 1, $A$, and 2), 18 anomalous arteries passed posterior to the aortic root and took a retroaortic course (Figure 1, B), and 1 anomalous artery extended around the front of the pulmonary artery and took a prepulmonic course.

Among the 50 patients with an interarterial course in our series, all patients had symptoms: chest pain (37/50), syncope (11/50), or dyspnea on exertion (11/50). Fifteen patients had significant coronary artery stenosis (stenosis rate $\geq 70 \%$ ), 14 mild or moderate coronary artery stenosis (stenosis rate $<70 \%$ ), and 21 had an absolutely normal coronary artery (no stenosis). Myocardial bridge was found in 8 of these 21 patients without any plaque within the coronary arteries.

Overall, conventional coronary angiography could not confidently identify the precise anomaly in 8 patients with anomalous arteries (Figure 1, $A$ and $C$ ).

In each case, DSCT-CA unequivocally demonstrated the origin of the anomalous coronary artery and its course in relation to the great vessels.

\section{DISCUSSION}

Coronary artery anomalies were classified as hemodynamically significant or hemodynamically insignificant. Hemodynamically significant anomalies include an anomalous interarterial course between the pulmonary artery and the aorta, an anomalous origin of either the left or right coronary artery from the pulmonary artery, occasional myocardial bridge, and coronary artery fistula.

It is widely documented that coronary insufficiency and sudden death are associated with some of these variants, specifically those that have a course that runs between the 2 great arteries. ${ }^{8}$ The exact pathophysiologic mechanisms that cause ischemia have not been determined. In our series, there were 13 symptomatic patients who had an anomalous interarterial course with no other concomitant obstructive coronary disease or myocardial bridge. The finding suggests that the important factor for presentation symptoms is the interarterial course itself, which is consistent with the study by Gulati and associates. ${ }^{8}$

Coronary artery anomalies are usually diagnosed during invasive coronary angiography. ${ }^{4}$ However, conventional coronary angiography sometimes is inadequate to delineate the precise course of the anomalous vessel because of a complex 3-dimensional geometry displayed in a relatively restricted 2-dimensional view. ${ }^{1}$ The low incidence of these 


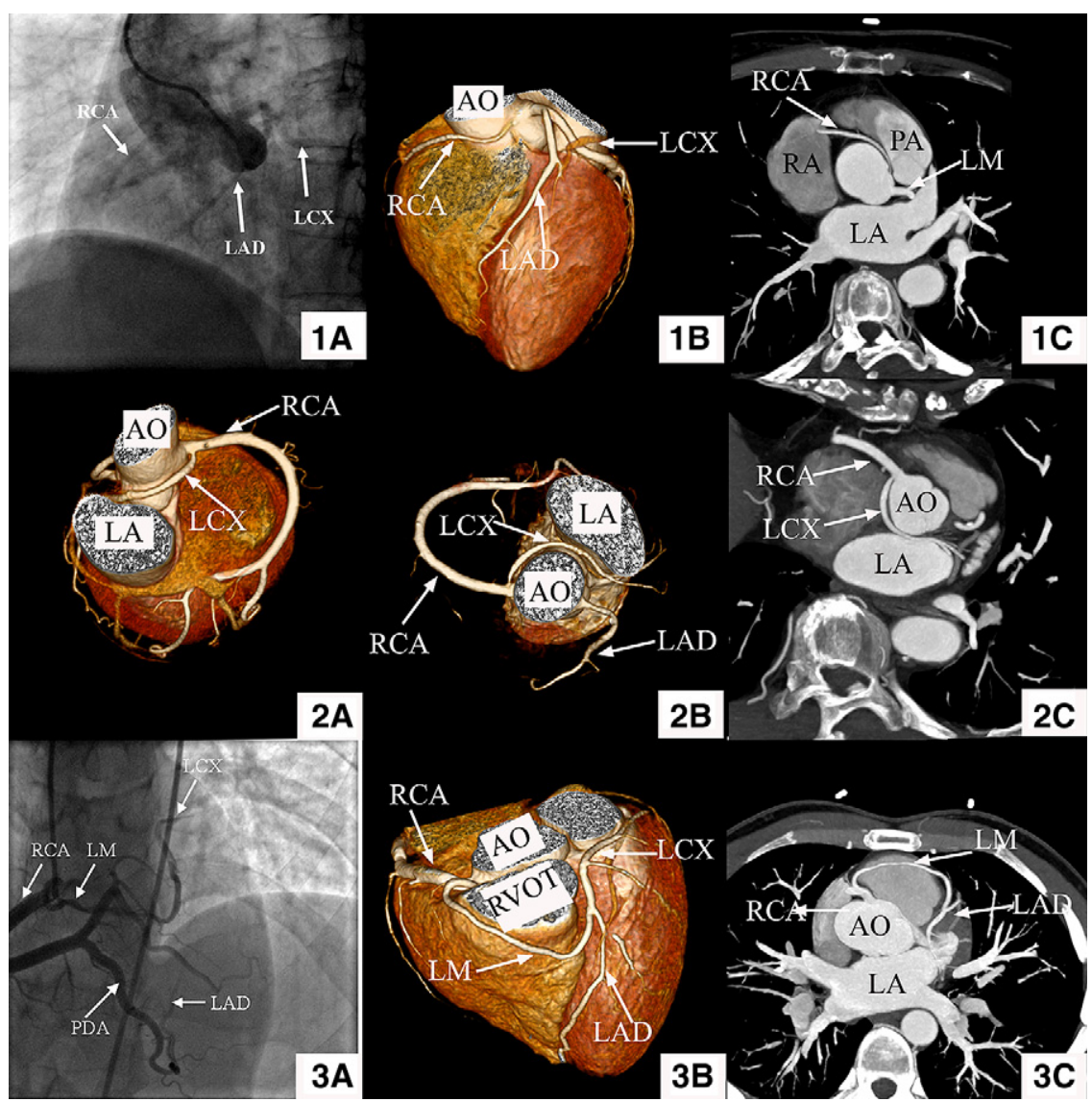

FIGURE 1. A, Images obtained in the patient with an anomalous origin of the RCA from the left sinus of Valsalva ( $L S V)$. $1 \mathrm{~A}$, Aortic root angiogram shows the anomalous origin of the RCA, but the exact location of the ostium is not identified. 1B and 1C, Three-dimensional volume-rendered and 10-mm maximum-intensity projection images show the RCA originating from the LSV. The slit-like ostium (black arrows) of the RCA is evident where the artery passes between the aortic root and the main pulmonary artery. B, Images obtained in the patient with an anomalous origin of an LCX from the right sinus of Valsalva $(R S V)$. 2A, Coronary angiogram shows the anomalous origin of the LM, but the exact origin of the anomalous coronary artery and its course in relationship to the great vessels are unequivocally demonstrated. $2 \mathrm{~B}$ and $2 \mathrm{C}$, Three-dimensional volume-rendered and 10-mm maximum-intensity projection images shows an anomalous origin for the LM, which arises from the RCA, courses anterior to the RVOT, and branches into the LAD and LCX. C, Images obtained in the patient with single coronary artery. 3A, 3B, and 3C, Three-dimensional volume-rendered and 10-mm maximum-intensity projection images show anomalous LCX originating from the RCA and passing posteriorly between the AO and LA to reach left atrioventricular groove. AO, Aorta; $R C A$, right coronary artery; $L A D$, left anterior descending coronary artery; $L C X$, left circumflex; $R A$, right atrium; $P A$, pulmonary artery; $L A$, left atrium; $L M$, left main coronary artery; $P D A$, patent ductus arteriosus; RVOT, right ventricular outflow tract.

anomalies also results in limited experience for many angiographers and causes a large percentage of coronary artery anomalies to be categorized incorrectly at invasive coronary angiography. ${ }^{9}$ Among 26 patients undergoing invasive coronary angiography in our study, the anomaly was incorrectly classified after an equivocal coronary angiogram in 8 patients (Figure 1, $A$ and $B$ ).

Noninvasive imaging techniques, including echocardiography, magnetic resonance (MR), electron-beam computed tomography, and MDCT, were introduced in the past few years and have proven to be reliable. ${ }^{10}$ Improvements in noninvasive diagnostic techniques have also increased the ability to easily and safely screen for the coronary congenital anomaly, leading to increased rates of diagnosis. ${ }^{11}$
Transesophageal echocardiography may clinically detect coronary anomalies, but this method is not totally noninvasive and is too costly for screening large populations. ${ }^{12}$ In our study, 1 patient with a complex coronary artery-pulmonary artery fistula with conus artery aneurysm received an incorrect diagnosis of thrombus in the right atrium (Figure 3, B). In a continuous series of 2388 transthoracic echocardiograms obtained in children, Davis and associates ${ }^{13}$ observed 1 false-negative echocardiographic finding followed by sudden death related to a coronary anomaly newly found at necropsy, ${ }^{13}$ raising doubts about this method's predictive value. ${ }^{12}$

Substantial investigative works have been performed with regard to the use of MR angiography in the evaluation of the coronary artery anomalies. ${ }^{14,15}$ MR angiography holds the 


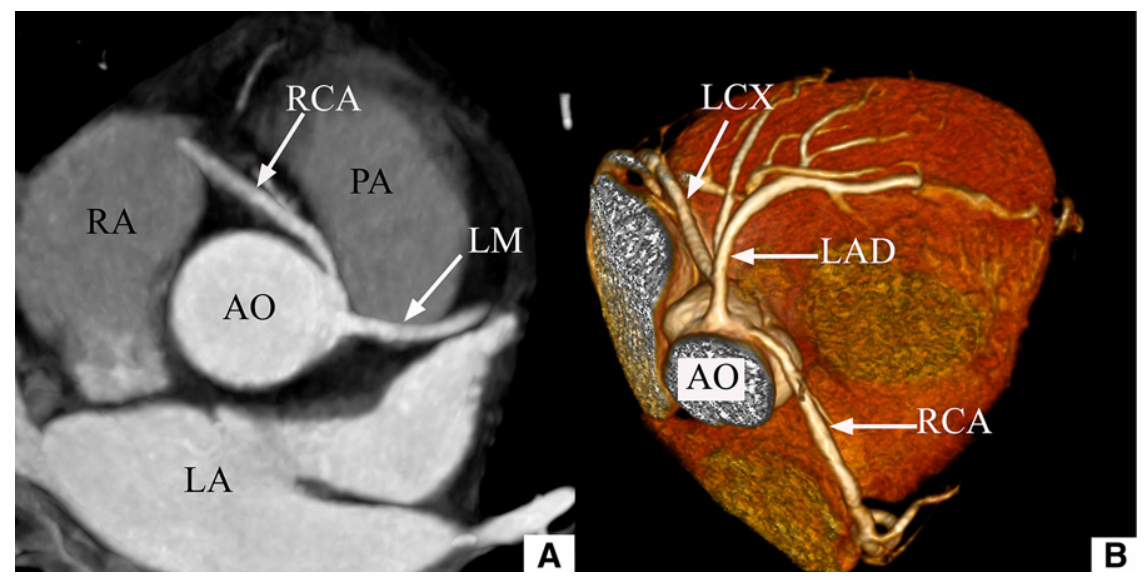

FIGURE 2. Images obtained in a 68-year-old man with a mean heart rate of 110 beats/min. A and B, Five-millimeter maximum-intensity projection and volume-rendered images clearly show the RCA originating from the LSV in the patient with a mean heart rate of 110 beats/min. For abbreviations, see Figure 1.

greatest appeal because it avoids radiation and iodinated contrast agents. As shown in several studies, ${ }^{14,15} \mathrm{MR}$ angiography provides an accurate assessment of the origin and proximal course of anomalous coronary arteries. Nevertheless, MR angiography has several limitations in the assessment of the coronary arteries. MR angiography can be limited by low spatial resolution and artifacts and can be technically challenging. Therefore, this technique is less helpful in evaluating fistulas and coronary origination outside the normal sinuses (eg, from a pulmonary artery). ${ }^{12}$

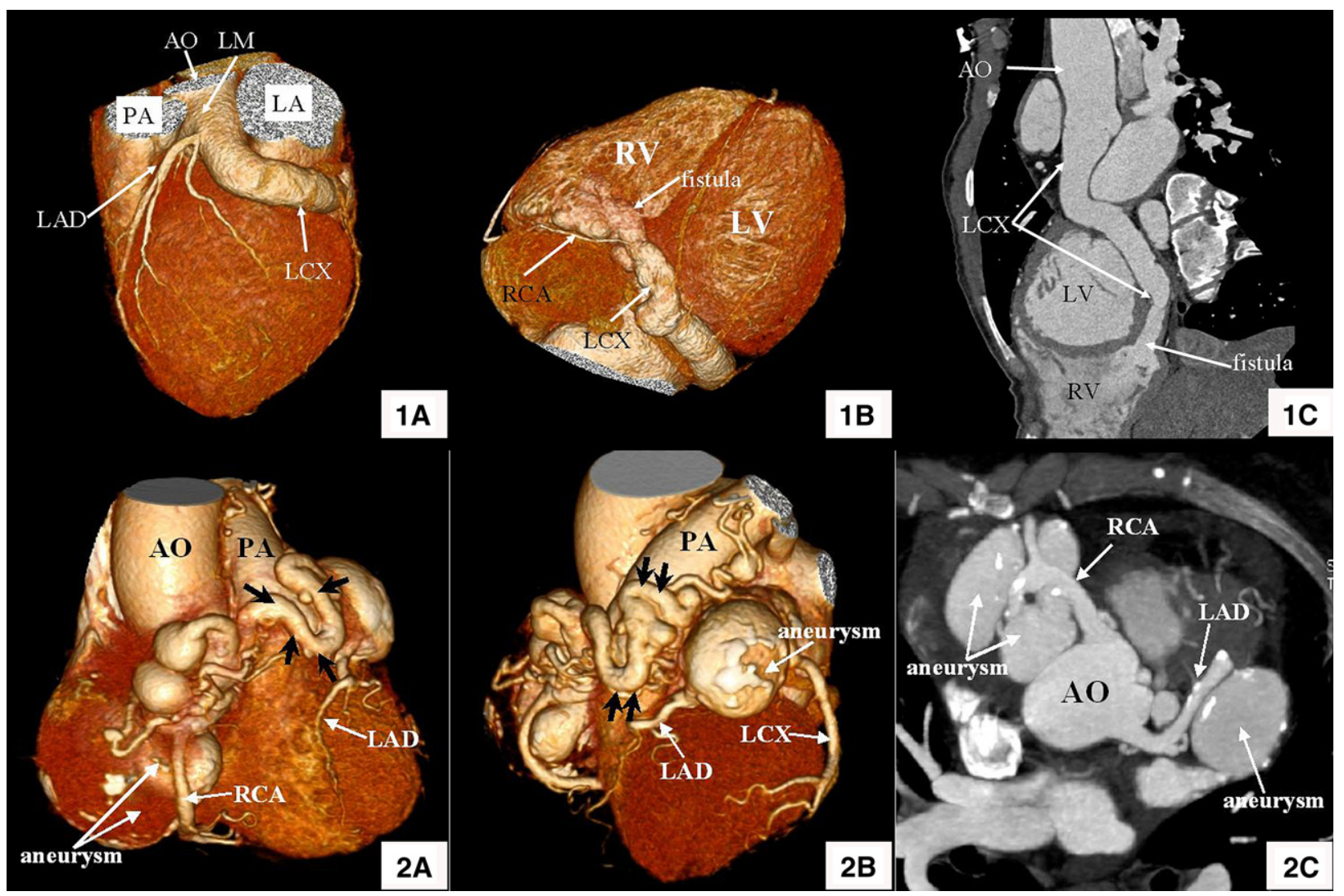

FIGURE 3. Images obtained in 2 patients with a coronary artery fistula. 1A, 1B, and 1C, Three-dimensional volume-rendered and 2-dimensional maximum-intensity projection images show the markedly enlarged and tortuous LCX coursing along the posterior cardiac surface. LM and LCX are markedly dilated while the LAD is of normal caliber. 2A, 2B, and 2C, Three-dimensional volume-rendered and 10-mm maximum-intensity projection images show an abnormal complex coronary artery to pulmonary artery fistula with LAD and RCA aneurysms. A plexus of tortuous vessels (black arrows) arising from the proximal RCA is clearly delineated. For abbreviations, see Figure 1. 
TABLE 1. Incidence of coronary anomalies and patterns, as observed in a continuous series of 12,415 DSCT-CA

\begin{tabular}{lr}
\hline \multicolumn{1}{c}{ Variable } & N $(\%)$ \\
\hline Coronary anomalies (total) & $124(1.02)$ \\
RCA from LSV (Figure 1, A) & $51(0.41)$ \\
LCX from RSV (Figure 1, B) & $17(0.14)$ \\
LM from RSV & $1(0.01)$ \\
Single coronary artery (Figure 1, C) & $4(0.03)$ \\
Anomalies associated with congenital & $1(0.01)$ \\
$\quad$ transposition of the great arteries & \\
Coronary artery fistulas (Figure 3) & $50(0.40)$ \\
\hline DSCT-CA, Dual-source computed tomography coronary angiography; $R C A$, right \\
coronary artery; $L S V$, left sinus of Valsalva; $L C X$, left circumflex; $R S V$, right sinus \\
of Valsalva; $L M$, left main coronary artery.
\end{tabular}

Furthermore, MR angiography cannot be performed for patients with pacemakers or defibrillating devices, and it may be difficult to perform for claustrophobic patients and patients with tachycardia or certain arrhythmias. ${ }^{1}$

Electron-beam computed tomography has a high temporal resolution of $100 \mathrm{~ms}$; however, its clinical application is limited by relatively poor z-axis resolution. ${ }^{1}$ Electrocardiographically gated MDCT systems were introduced in 1999. This is a most significant development for evaluation of the coronary artery disease. It enables rapid coverage of the coronary territory in a single breath hold and improved longitudinal resolution, approaching the ideal of "isotropic" spatial resolution. ${ }^{16}$ It has been reported that MDCT is superior to conventional angiography in delineating the ostial origin and proximal path of the anomalous coronary artery. ${ }^{17}$ However, MDCT imaging can be limited by motion artifacts caused by limited temporal resolution in patients with high heart rates as well as patients with arrhythmia. Therefore, beta-blocker administration is routinely used to reduce the heart rate, minimizing residual heart motion in all patients with a heart rate of more than 65 beats $/ \mathrm{min}$. Nevertheless, some patients with chronic obstructive pulmonary disease, bronchial asthma, second-and third-degree heart block, and severe peripheral vascular disease have contraindications to the use of beta-blocker drugs, and administration of beta-blocker drugs to patients scheduled for MDCT-CA might be logistically difficult.

With the recently introduced DSCT, which mainly improves the temporal resolutions, it is now possible and practical to have high-quality cardiac imaging without beta-blocker premedication even in patient with high heart rates. ${ }^{6,7}$ The DSCT system is equipped with 2 x-ray tubes and 2 corresponding detectors, which are mounted onto the rotating gantry with an angular offset of $90^{\circ}$. The temporal resolution is as high as $82.5 \mathrm{~ms}$. The better temporal resolution and the ability to choose the optimal cardiac phase result in a near cessation of physiologic motion. As the spatial resolution of data collected is isotropic, images can be reviewed by 2-dimensional MPR
TABLE 2. Origin and drainage sites of coronary artery fistulas in $\mathbf{5 0}$ patients (n)

\begin{tabular}{lccccc}
\hline \multicolumn{1}{c}{ Origin } & RV & LV & PA & GCV & Total* \\
\hline RCA & 2 & 0 & 8 & 1 & $11(22.0)$ \\
LAD & 1 & 0 & 26 & 0 & $27(54.0)$ \\
LCX & 1 & 1 & 0 & 0 & $2(4.0)$ \\
DA & 0 & 1 & 0 & 0 & $1(2.0)$ \\
RCA+LAD & 0 & 0 & 9 & 0 & $9(18.0)$ \\
Total ${ }^{*}$ & $4(8.0)$ & $2(4.0)$ & $43(86.0)$ & $1(2.0)$ & \\
\hline
\end{tabular}

$R V$, Right ventricle; $L V$, left ventricle; $P A$, pulmonary artery; $G C V$, great cardiac vein; $R C A$, right coronary artery; $L A D$, left descending coronary artery; $L C X$, left circumflex; $D A$, diagonal branch of coronary artery. *Values in parentheses are percentages of overall total.

and 3-dimensional reconstruction techniques including VR and MIP. ${ }^{18}$ The 3-dimensional reconstruction with viewing at an unlimited angle allows us to demonstrate anomalous vessels at the best projection, without repeated radiation exposure and an additional contrast load, and makes assessment of the size and exact location of the anomalous vessel feasible. Although these images were not crucial in the diagnosis of the anomaly, they were valuable for depicting the relationships among the coronary vessel, great vessels, and ventricles. Such images could be helpful for planning future cardiovascular intervention and give the surgeons a better understanding of the complex anatomy before operation. ${ }^{1,18}$ In our study, DSCT-CA unequivocally demonstrated the origin of the anomalous coronary artery and its course in relation to the great vessels in all cases, even in 1 patient with a mean heart rate of 110 beats/min (Figure 2). In addition, data obtained during DSCT-CA are often used to evaluate obstructive coronary disease, as well as cardiovascular function. ${ }^{19,20}$ Among the 50 patients with an interarterial course in our series, 15 patients had significant coronary artery stenosis (stenosis rate $\geq 70 \%$ ). Such information was also useful to help the surgeons to make the decision of which surgical techniques to use to repair the anomaly.

DSCT-CA also has its own drawbacks. Allergy, renal, or cardiac insufficiency may prevent the use of intravenous contrast material in some patients. MR angiography is considered as a supplement in these patients. Another drawback is the high effective radiation dose to which a patient undergoing DSCT angiography is exposed. In our study, the mean effective radiation dose was $13.6 \mathrm{mSv}$. By comparison, the mean effective radiation dose with diagnostic coronary arteriography is 2.1 to $2.5 \mathrm{mSv}$ as reported by Hunold and associates. ${ }^{21}$ Fortunately, investigators have shown that DSCT has the potential to reduce radiation dose exposure to as low as $3.8 \mathrm{mSv}$ if the optimized and individual scan protocol is used. ${ }^{22}$ With the recent advent of second-generation DSCT equipped with two 128-slice acquisition detectors, the prospectively electrocardiographically gated step-andshoot and the high-pitch mode data acquisition significantly lowers the radiation dose to less than $1 \mathrm{mSv}^{23}$ 


\section{Limitations}

We acknowledge the following study limitations. The most notable limitation is lack of a reference standard technique to prove that our DSCT-CA findings were correct. The direct visualization at surgery was considered to be the reference standard. Unfortunately, this "confirmatory" information was obtained in only 3 patients with coronary anomalies who underwent surgery. It is also reported that even surgery cannot be considered a consistently reliable standard reference technique because the anomalous coronary course is often obscured by epicardial fat. ${ }^{1}$ Another limitation is incomplete statistics of coronary anomalies. Myocardial bridges, duplication of arteries, high takeoff, and multiple ostia of coronary arteries were not counted in our study. One of the reasons is that, at the time of writing, there are no clear diagnostic criteria and no unified classification of the multiple variations. The greatest confusion in this regard is about myocardial bridges. Angelini ${ }^{24}$ suggests that they may be a normal variant.

Coronary arterial anomalies should be suspected in any young patient with symptoms of angina pectoris, nearfatal arrhythmias, or recurrent syncope. ${ }^{25}$ DSCT-CA is a reliable noninvasive imaging tool in the delineation of coronary arterial anomalies, and it is superior to conventional invasive coronary angiography in delineating the origin and course of an anomalous coronary artery.

\section{References}

1. Datta J, White CS, Gilkeson RC, Meyer CA, Kansal S, Jani ML, et al. Anomalous coronary arteries in adults: depiction at multi-detector row CT angiography. $R a$ diology. 2005;235:812-8.

2. Maron BJ. Sudden death in young athletes. N Engl J Med. 2003;349:1064-75.

3. Maron BJ, Carney KP, Lever HM, Lewis JF, Barac I, Casey SA, et al. Relationship of race to sudden cardiac death in competitive athletes with hypertrophic cardiomyopathy. J Am Coll Cardiol. 2003;41:974-80.

4. Kim SY, Seo JB, Do KH, Heo JN, Lee JS, Song JW, et al. Coronary artery anomalies: classification and ECG-gated multi-detector row CT findings with angiographic correlation. Radiographics. 2006;26:317-33; discussion 33-4.

5. Pannu HK, Flohr TG, Corl FM, Fishman EK. Current concepts in multi-detector row CT evaluation of the coronary arteries: principles, techniques, and anatomy. Radiographics. 2003;23 Spec No:S111-25.

6. Achenbach S, Ropers D, Kuettner A, Flohr T, Ohnesorge B, Bruder H, et al. Contrast-enhanced coronary artery visualization by dual-source computed tomography—initial experience. Eur J Radiol. 2006;57:331-5.
7. Brodoefel H, Burgstahler C, Tsiflikas I, Reimann A, Schroeder S, Claussen CD, et al. Dual-source CT: effect of heart rate, heart rate variability, and calcification on image quality and diagnostic accuracy. Radiology. 2008;247:346-55.

8. Gulati R, Reddy VM, Culbertson C, Helton G, Suleman S, Reinhartz O, et al. Surgical management of coronary artery arising from the wrong coronary sinus, using standard and novel approaches. J Thorac Cardiovasc Surg. 2007;134: 1171-8.

9. Ishikawa T, Brandt PW. Anomalous origin of the left main coronary artery from the right anterior aortic sinus: angiographic definition of anomalous course. Am J Cardiol. 1985;55:770-6.

10. Ropers D, Moshage W, Daniel WG, Jessl J, Gottwik M, Achenbach S. Visualization of coronary artery anomalies and their anatomic course by contrastenhanced electron beam tomography and three-dimensional reconstruction. Am J Cardiol. 2001;87:193-7.

11. Fedoruk LM, Kern JA, Peeler BB, Kron IL. Anomalous origin of the right coronary artery: right internal thoracic artery to right coronary artery bypass is not the answer. J Thorac Cardiovasc Surg. 2007;133:456-60.

12. Angelini P, Velasco JA, Flamm S. Coronary anomalies: incidence, pathophysiology, and clinical relevance. Circulation. 2002;105:2449-54.

13. Davis JA, Cecchin F, Jones TK, Portman MA. Major coronary artery anomalies in a pediatric population: incidence and clinical importance. J Am Coll Cardiol. 2001;37:593-7.

14. Bunce NH, Lorenz CH, Keegan J, Lesser J, Reyes EM, Firmin DN, et al. Coronary artery anomalies: assessment with free-breathing three-dimensional coronary MR angiography. Radiology. 2003;227:201-8.

15. Gharib AM, Ho VB, Rosing DR, Herzka DA, Stuber M, Arai AE, et al. Coronary artery anomalies and variants: technical feasibility of assessment with coronary MR angiography at 3 T. Radiology. 2008;247:220-7.

16. Schoepf UJ, Becker CR, Ohnesorge BM, Yucel EK. CT of coronary artery disease. Radiology. 2004;232:18-37.

17. Shi H, Aschoff AJ, Brambs HJ, Hoffmann MH. Multislice CT imaging of anomalous coronary arteries. Eur Radiol. 2004;14:2172-81.

18. Chan MS, Chan IY, Fung KH, Lee G, Tsui KL, Leung TC. Demonstration of complex coronary-pulmonary artery fistula by MDCT and correlation with coronary angiography. AJR Am J Roentgenol. 2005;184(3 Suppl):S28-32.

19. Brodoefel H, Kramer U, Reimann A, Burgstahler C, Schroeder S, Kopp A, et al Dual-source CT with improved temporal resolution in assessment of left ventricular function: a pilot study. AJR Am J Roentgenol. 2007;189:1064-70.

20. Oncel D, Oncel G, Tastan A, Tamci B. Evaluation of coronary stent patency and in-stent restenosis with dual-source CT coronary angiography without heart rate control. AJR Am J Roentgenol. 2008;191:56-63.

21. Hunold P, Vogt FM, Schmermund A, Debatin JF, Kerkhoff G, Budde T, et al. Radiation exposure during cardiac CT: effective doses at multi-detector row $\mathrm{CT}$ and electron-beam CT. Radiology. 2003;226:145-52.

22. Rixe J, Conradi G, Rolf A, Schmermund A, Magedanz A, Erkapic D, et al. Radiation dose exposure of computed tomography coronary angiography: comparison of dual-source, 16-slice and 64-slice CT. Heart. 2009;95:1337-42.

23. Alkadhi H, Stolzmann P, Desbiolles L, Baumueller S, Goetti R, Plass A, et al. Low-dose, 128-slice, dual-source CT coronary angiography: accuracy and radiation dose of the high-pitch and the step-and-shoot mode. Heart. 2010;96:933-8.

24. Angelini P. Coronary artery anomalies: an entity in search of an identity. Circulation. 2007;115:1296-305.

25. Basso C, Maron BJ, Corrado D, Thiene G. Clinical profile of congenital coronary artery anomalies with origin from the wrong aortic sinus leading to sudden death in young competitive athletes. J Am Coll Cardiol. 2000;35:1493-501. 